\title{
Relationship between SYNTAX score and Trans-Atlantic Inter-Society Consensus II classification in patients undergoing diagnostic angiography
}

\author{
Hakan Erkan¹, Bülent Vatan², Mustafa Tarık Ağaç ${ }^{1}$, Levent Korkmaz ${ }^{1}$, Merve Erkan ${ }^{3}$, Abdulkadir Kırıșs $^{1}$, \\ Zeydin Acar ${ }^{1}$, Șükrü Çelik ${ }^{1}$
}

${ }^{1}$ Ahi Evren Cardiovascular and Thoracic Surgery Hospital, Trabzon, Turkey

${ }^{2}$ Sakarya Research and Training Hospital, Sakarya, Turkey

${ }^{3}$ Karadeniz Technical University Hospital, Trabzon, Turkey

Postep Kardiol Inter 2013; 9, 4 (34): 344-347

DOI: $10.5114 /$ pwki.2013.38863

\begin{abstract}
A bstract
Introduction: Peripheral artery disease (PAD) is a marker of systemic atherosclerosis and most patients with PAD also have concomitant coronary artery disease (CAD).

Aim: There are no published data investigating the relationship between PAD and CAD complexity assessed by a well-accepted classification system such as the SYNTAX score (SS) or Trans-Atlantic Inter-Society Consensus II (TASC II).

Material and methods: The study population consisted of 72 patients who underwent coronary angiography for the assessment of CAD. At the same session, peripheral angiography was performed in cases of suspected PAD. A coronary lesion was defined as significant if it caused a $50 \%$ reduction of the luminal diameter by visual estimation in vessels $\geq 1.5 \mathrm{~mm}$. The SYNTAX score was computed by dedicated software.

Results: Patients with peripheral artery disease were divided into four groups according to the Trans-Atlantic Inter-Society Consensus II classification. Numbers of patients with peripheral artery disease classified as A, B, C, and D by the Trans-Atlantic Inter-Society Consensus II classification were $27,16,18$ and 11 , respectively. SYNTAX scores for each group from A to D were $10 \pm 9$, $11 \pm 10,24 \pm 13$ and $27 \pm 12$, respectively; $p$ for trend $<0.001$.

Conclusions: Higher Trans-Atlantic Inter-Society Consensus II classification is associated with higher SYNTAX score in patients who underwent coronary and peripheral diagnostic angiography. It may suggest that arterial atherosclerotic disease complexity is a systemic panvascular phenomenon.
\end{abstract}

Key words: coronary artery, peripheral artery, TASC II, SYNTAX.

\section{Introduction}

The SYNTAX score is a comprehensive angiographic scoring system that is derived entirely from the coronary anatomy and lesion characteristics and widely accepted as a coronary artery disease (CAD) complexity marker [1-3]. Peripheral arterial disease (PAD) is a well-established risk factor for cardiovascular disease. In previous studies, PAD has been associated with an increased risk of cardiovascular and all-cause mortality and incidence of CAD $[4,5]$. This is believed to be due to the higher prevalence of cardiac risk factors among patients with PAD [6]. Our hypothesis was that increased cardiovascular events in patients with PAD may be due, at least in part, to the higher prevalence of complex coronary lesions.
Aim

Although an association between PAD and CAD severity has been demonstrated, there is no study investigating the relationship between $C A D$ complexity assessed by the SYNTAX score (SS) and PAD complexity determined by the Trans-Atlantic Inter-Society Consensus II (TASC II) classification.

\section{Material and methods \\ Patients}

In this retrospective study there were enrolled $72 \mathrm{pa}$ tients who underwent elective coronary angiography and needed peripheral angiography because of suspected

\section{Corresponding author:}

Hakan Erkan, MD, Ahi Evren Cardiovascular and Thoracic Surgery Hospital, Camlik St, 61300 Trabzon, Turkey, tel.: +90 5543173588,

e-mail: drhakanerkan@hotmail.com

Received: 7.02.2013, accepted: 20.06.2013. 
PAD. Informed consent was obtained for coronary and peripheral angiography in all patients. The study protocol was approved by the local ethics committee. Angiography indications in patients with suspected PAD were as follows: physician recommendation based on patients' clinical history before coronary angiography, and difficulties in puncturing femoral arteries and advancing the guide wire or diagnostic catheters during angiography. Peripheral angiography was performed transfemorally with a 6-Fr pigtail catheter. For evaluation of both lower extremities, the catheter tip was positioned above the aorta-iliac bifurcation. TASC II score analysis was performed on bilateral aorta-iliac arterial segments. Patients were excluded if they had undergone any previous peripheral or coronary artery revascularizations.

\section{Angiography and SYNTAX score}

Coronary angiography and aortography were analyzed independently by 2 experienced interventional cardiologists. In cases of disagreement, the decision of a third observer was obtained and the final decision was made by consensus. Each coronary lesion producing $50 \%$ diameter stenosis in vessels of diameter $1.5 \mathrm{~mm}$ was scored separately and added together to provide the overall SS, which was calculated using the SS score algorithm [3].

\section{Statistical analysis}

Continuous variables were expressed as mean \pm standard deviation (SD) and categorical variables were expressed as percentage. An analysis of normality of the continuous variables was performed with the Kolmogorov-Smirnov test. Comparison of the SS among PAD subgroups was made using one-way analysis of variance (ANOVA) with post hoc Tukey test. Statistical analysis was performed using SPSS 14.0 and a value of $p$ of 0.05 (2-tailed) was considered significant.

\section{Results}

Clinical and demographic characteristics of the study population are given in Table 1. Angiographic data of patients are provided in Table 2. Seventy-two patients were divided into 4 groups by TASC II classification. Mean SS was $17 \pm 13$. Numbers of patients with PAD of A, B, C, and D by the TASC II classification were 27, 16, 18 and 11 , respectively. SSs for each group from $A$ to $D$ were $10 \pm 9,11 \pm 10,24 \pm 13$ and $27 \pm 12$, respectively; $p$ for trend $<0.001)$ (Figure 1). There was no significant difference of SS for groups A and B ( $p=0.99)$. Even though there was a trend for higher SS for group D compared to group C, it did not reach statistical significance $(p=0.8)$. Value of $p$ for groups $B$ and $C$ was 0.006 . For groups $B$ and $D$, $p$ value was 0.001
Table 1. Baseline and laboratory characteristics of study population $(n=72)$

\begin{tabular}{lc} 
Parameter & Value \\
\hline Age, mean \pm SD & $63 \pm 9$ \\
\hline Male gender, $n(\%)$ & $49(68)$ \\
\hline Smoking, $n(\%)$ & $38(53)$ \\
\hline Diabetes, $n(\%)$ & $14(20)$ \\
\hline Hypertension, $n(\%)$ & $34(47)$ \\
\hline Dyslipidemia, $n(\%)$ & $32(44)$ \\
\hline Total cholesterol, mean \pm SD & $207 \pm 37$ \\
\hline Glucose, mean \pm SD & $113 \pm 38$ \\
\hline LDL, mean \pm SD & $135 \pm 32$ \\
\hline HDL, mean \pm SD & $36 \pm 9$ \\
\hline Triglyceride, mean \pm SD & $194 \pm 90$ \\
\hline Creatin, mean \pm SD & $1.1 \pm 0.21$
\end{tabular}

$L D L$ - low density cholesterol, $H D L$ - high density cholesterol

Table 2. Angiographic characteristics of study population $(n=72)$

\begin{tabular}{ll}
\hline Parameter & Value \\
\hline Number of diseased coronary vessels, $n(\%)$ & \\
\hline One vessel & $21(29)$ \\
\hline Two vessels & $24(33)$ \\
\hline Three vessels & $27(38)$ \\
\hline Syntax score, mean \pm SD & $10 \pm 9$ \\
\hline Type A & $11 \pm 10$ \\
\hline Type B & $24 \pm 13$ \\
\hline Type C & $27 \pm 12$ \\
\hline Type D & $27(38)$ \\
\hline Classification of peripheral artery disease, $n(\%)$ & $16(22)$ \\
\hline Type A & $18(25)$ \\
\hline Type B & $11(15)$ \\
\hline Type C
\end{tabular}

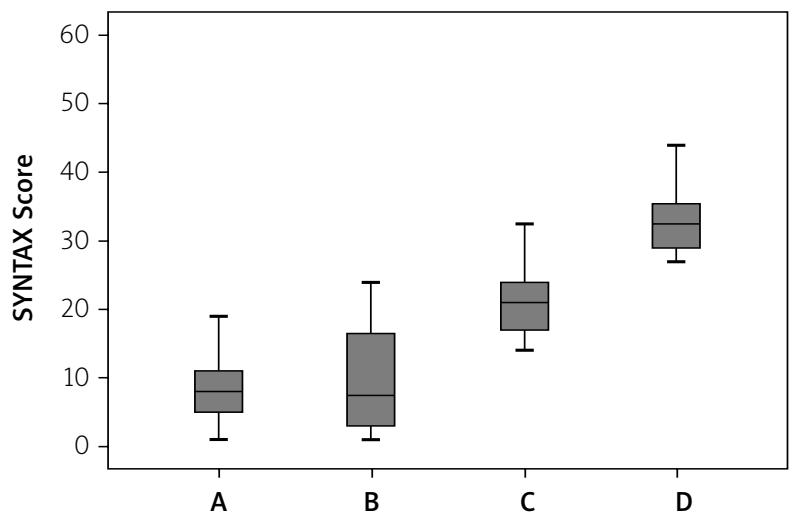

Fig. 1. Syntax score in patients with different TASC II groups 


\section{Discussion}

In the present study, we have demonstrated a significant association between coronary and peripheral artery disease complexity using SYNTAX score and Trans-Atlantic Inter-Society Consensus II classifications. This may suggest that complex arterial disease in a region should be regarded as a sign of complex panvascular disease.

Peripheral arterial disease is a marker of systemic atherosclerosis. Previous studies have shown that patients with symptomatic or asymptomatic PAD often present with coronary atherosclerosis and are at increased risk for adverse cardiovascular events $[4,5]$. The ankle brachial in$\operatorname{dex}(A B I)$ is a noninvasive marker of presence and severity of PAD. It is significantly correlated with the number of occluded vessels in acute coronary syndrome patients [7]. Also, $A B I$ is a statistically significant independent predictor for lesion site, complex stenotic lesions and lesion morphology in patients with angina pectoris suspected for CAD [8]. The presence of an abnormal $A B I(\leq 0.9$ or $>1.4)$ was associated with an increased risk of multivessel disease [9]. There was a significant association between degree of PAD and higher prevalence of 3- or 4-vessel CAD and lower prevalence of 1-vessel CAD [10]. Furthermore, $A B I$ is a predictor of CAD severity determined by Gensini score [11].

Our study is quite different from those mentioned above, for several reasons. First of all, none of them used the SYNTAX score as a CAD complexity marker. Secondly, most of them regarded $A B I$, not TASC II classification, as a PAD severity marker. In fact, $A B I$ is a functional, not an anatomical marker. Also a high level of specificity (83.3$99.0 \%)$ and accuracy (72.1-89.2\%) was reported for an $\mathrm{ABI} \leq 0.90$ in detecting $\geq 50 \%$ stenosis, but there were different levels of sensitivity (15-79\%) [12]. Many clinical and vascular factors may contribute to the sensitivity of $A B I$, including patients' age, ethnicity, and health status, as well as other factors. Sensitivity of $A B I$ for detecting PAD is especially low for elderly and diabetic patients $[13,14]$. More calcified arteries may not be compressible and reveal a higher $A B I$ value and underestimate severity of PAD [15]. In addition, the elevated ABI values in spite of stenosis could be due to collateral circulation, which maintains blood flow to the lower limb beyond the obstruction [12]. Therefore, $A B I$ should not be considered as good a PAD complexity marker as direct visualization by angiography.

There are several important limitations of our study. The major limitation is related to our patient selection protocol, which possesses some inherent biases. Our study was retrospective and only included cases of suspected PAD patients. Given that a high proportion of PAD patients are asymptomatic during coronary angiography [16], a large prospective study of examination of both the coronary and peripheral artery during the same session by angiography is needed to confirm our results. Other limitations are the small sample size and cross-sectional nature of the study.

\section{Conclusions}

We have shown for the first time a significant association between coronary and peripheral artery disease complexity using SYNTAX score and Trans-Atlantic InterSociety Consensus II classifications. Our results suggest that vascular complexity may be a panvascular phenomenon and increased vascular complexity may be caused by both local and systemic factors and lead to increased cardiovascular events in this patient population. Further studies are required to elucidate the 'panvascular complexity' concept.

\section{References}

1. Sianos G, Morel MA, Kappetein AP, et al. The SYNTAX score: an angiographic tool grading the complexity of coronary artery disease. Eurointervention 2005; 1: 219-227.

2. Serruys P, Onuma Y, Garg S, et al. Assessment of the SYNTAX score in the Syntax study. Eurointervention 2009; 5: 50-56.

3. SYNTAX Working Group. SYNTAX score calculator. http://www. syntaxscore.com. Accessed September 15, 2011.

4. Megnien JL, Simon A, Gariepy J, et al. Preclinical changes of extracoronary arterial structures as indicators of coronary atherosclerosis in men. J Hypertens 1998; 16: 157-163.

5. Khoury Z, Schwartz R, Gottlieb S, et al. Relation of coronary artery disease to atherosclerotic disease in the aorta, carotid and femoral arteries evaluated by ultrasound. Am J Cardiol 1997; 80: 1492-1433.

6. Willens HJ, Davis W, Herrington DM, et al. Relationship of peripheral arterial compliance and standard cardiovascular risk factors. Vasc Endovascular Surg 2003; 37: 197-206.

7. Chen CC, Hung KC, Hsieh IC, et al. Association between peripheral vascular disease indexes and the numbers of vessels obstructed in patients with coronary artery disease. Am J Med Sci 2012; 343: 52-55.

8. Chang ST, Chen CL, Chu CM, et al. Ankle-arm index as a predictor of lesion morphology and risk classification for coronary artery disease undergoing angioplasty. Int J Cardiol 2006; 113: 385-390.

9. Morillas P, Quiles J, Cordero A, et al. Prevalence of Peripheral Arterial Disease in Patients With Acute Coronary Syndrome (PAMISCA) Investigators. Prevalence of Peripheral Arterial Disease in Patients With Acute Coronary Syndrome (PAMISCA) investigators. Impact of clinical and subclinical peripheral arterial disease in mid-term prognosis of patients with acute coronary syndrome. Am J Cardiol 2009; 104: 1494-1498.

10. Sukhija R, Aronow WS, Yalamanchili K, et al. Association of ankle-brachial index with severity of angiographic coronary artery disease in patients with peripheral arterial disease and coronary artery disease. Cardiology 2005; 103: 158-160.

11. Papamichael CM, Lekakis JP, Stamatelopoulos KS, et al. Ankle-brachial index as a predictor of the extent of coronary atherosclerosis and cardiovascular events in patients with coronary artery disease. Am J Cardiol 2000; 86: 615-618.

12. Dachun Xu, Jue Li, Liling Zou, et al. Sensitivity and specificity of the ankle-brachial index to diagnose peripheral artery disease: a structured review. Vasc Med 2010; 15: 361-369.

13. Parameswaran GI, Brand K, Dolan J. Pulse oximetry as a potential screening tool for lower extremity arterial disease in asymptomatic patients with diabetes mellitus. Arch Intern Med 2005; 165: 442-446. 
14. Williams DT, Harding KG, Price P. An evaluation of the efficacy of methods used in screening for lower-limb arterial disease in diabetes. Diabetes Care 2005; 28: 2206-2210.

15. Wikstrom J, Hansen T, Johansson L, et al. Ankle brachial index $<0.9$ underestimates the prevalence of peripheral artery occlusive disease assessed with whole-body magnetic resonance angiography in the elderly. Acta Radiol 2008; 49: 143-149.

16. Moussa ID, Jaff MR, Mehran R, et al. Prevalence and prediction of previously unrecognized peripheral arterial disease in patients with coronary artery disease: the Peripheral Arterial Disease in Interventional Patients Study Catheter Cardiovasc Interv 2009; 73: 719-724. 